\title{
Cyclic hydrostatic pressure promotes a stable cartilage phenotype and enhances the functional development of cartilaginous grafts engineered using multipotent stromal cells isolated from bone marrow and infrapatellar fat pad
}

\author{
S.F. Carroll ${ }^{\mathrm{a}, \mathrm{b}}$, C.T. Buckley ${ }^{\mathrm{a}, \mathrm{b}}$, D.J. Kelly ${ }^{\mathrm{a}, \mathrm{b}, \mathrm{c}, *}$ \\ a Trinity Centre for Bioengineering, Trinity Biomedical Sciences Institute, Trinity College Dublin, Ireland \\ ${ }^{\mathrm{b}}$ Department of Mechanical and Manufacturing Engineering, School of Engineering, Trinity College Dublin, Dublin 2, Ireland \\ c Advanced Materials and BioEngineering Research (AMBER) Centre, Trinity College Dublin, Ireland
}

\section{A R T I C L E I N F O}

Article history:

Accepted 6 December 2013

\section{Keywords:}

Cartilage repair

Functional tissue engineering

Mesenchymal stem cell

Multipotent stromal cell

Hydrostatic pressure

Chondrogenesis

Hypertrophy

Mechanical stimulation

\begin{abstract}
A B S T R A C T
The objective of this study was to investigate how joint specific biomechanical loading influences the functional development and phenotypic stability of cartilage grafts engineered in vitro using stem/progenitor cells isolated from different source tissues. Porcine bone marrow derived multipotent stromal cells (BMSCs) and infrapatellar fat pad derived multipotent stromal cells (FPSCs) were seeded in agarose hydrogels and cultured in chondrogenic medium, while simultaneously subjected to $10 \mathrm{MPa}$ of cyclic hydrostatic pressure (HP). To mimic the endochondral phenotype observed in vivo with cartilaginous tissues engineered using BMSCs, the culture media was additionally supplemented with hypertrophic factors, while the loss of phenotype observed in vivo with FPSCs was induced by withdrawing transforming growth factor (TGF)- $\beta 3$ from the media. The application of HP was found to enhance the functional development of cartilaginous tissues engineered using both BMSCs and FPSCs. In addition, HP was found to suppress calcification of tissues engineered using BMSCs cultured in chondrogenic conditions and acted to maintain a chondrogenic phenotype in cartilaginous grafts engineered using FPSCs. The results of this study point to the importance of in vivo specific mechanical cues for determining the terminal phenotype of chondrogenically primed multipotent stromal cells. Furthermore, demonstrating that stem or progenitor cells will appropriately differentiate in response to such biophysical cues might also be considered as an additional functional assay for evaluating their therapeutic potential.
\end{abstract}

(c) 2013 Elsevier Ltd. All rights reserved.

\section{Introduction}

Articular cartilage possesses a limited capacity for self-repair. Current clinical therapies for cartilage defect repair such as autologous chondrocyte implantation suffer from significant drawbacks, such as donor site morbidity and dedifferentiation of chondrocytes during monolayer expansion. Mesenchymal stem cells possess the ability to proliferate extensively ex vivo whilst maintaining their multipotent differentiation capabilities (Bruder et al., 1997; Kadiyala et al., 1997) and can be induced to differentiate towards a chondrogenic lineage (Caplan, 1991; Johnstone et al., 1998; Mackay et al., 1998; Pittenger et al., 1999; Sheehy et al., 2012a; Vinardell et al., 2012b), making them an attractive alternative cell type for cartilage repair strategies. Multipotent cells can be isolated from numerous different tissues. There are,

\footnotetext{
* Corresponding author at: Department of Mechanical and Manufacturing Engineering, School of Engineering, Trinity College Dublin, Dublin 2, Ireland. Tel.: +353189639 47; fax: +353167955 54 .

E-mail address: kellyd9@tcd.ie (D.J. Kelly).
}

however, significant differences in their properties depending on cell source, with joint tissue derived stem cells having a gene expression profile closer to that of chondrocytes when compared to extra-articular tissue derived stem cells (Segawa et al., 2009). There is also evidence to suggest that joint tissue derived multipotent stromal cells, such as those isolated from the infrapatellar fat pad (IFP) or synovium, may possess a superior chondrogenic potential to those derived from alternative sources (Sakaguchi et al., 2005; Vinardell et al., 2012b). In spite of this, the in vivo phenotypic stability of cartilaginous tissues engineered using such cells, irrespective of their origin, is by no means assured (De Bari et al., 2004; Pelttari et al., 2006; Hennig et al., 2007; Dickhut et al., 2008). Previous studies have shown that following in vivo subcutaneous implantation, IFP derived multipotent stromal cells (FPSCs) tend to undergo fibrous dedifferentiation or resorption, as evidenced by increased collagen type I and reduced sulphated glycosaminoglycan (sGAG) content, whereas bone marrow derived multipotent stromal cells (BMSCs) tend to follow an endochondral pathway, with increased mineralisation and vascularisation of the engineered tissue (Pelttari et al., 2006; Vinardell et al., 2012b). 
This highlights the potential challenges associated with using any such cell type for successfully regenerating articular cartilage.

Within the orthotopic environment of a full thickness cartilage defect, it has been demonstrated that hypertrophy of implanted multipotent stromal cells (MSCs) is restricted to a region of the regenerating tissue close to the bone, demonstrating that in vivo signalling molecules and biomechanical stimuli play a key role in regulating the chondrogenic phenotypic (Steck et al., 2009). Identifying how such in vivo specific environmental cues regulate the terminal phenotype of MSCs may play a key role in realising their full therapeutic potential. Mechanical signals, such as hydrostatic pressure (HP), are a key component of the in vivo joint environment and have been shown to play a significant role in regulating the chondrogenic differentiation of mesenchymal stem cells. Previous studies have shown varying and occasionally conflicting results (Parkkinen et al, 1993; Carver and Heath, 1999; Suh et al., 1999; Carver and Heath, 2000; Jortikka et al., 2000; Smith et al., 2000, 2005; Hu and Athanasiou, 2006; Finger et al., 2007; Ogawa et al., 2009; Meyer et al., 2011; Liu et al., 2012; Maxson and Burg, 2012; Puetzer et al., 2012; Safshekan et al., 2012; Steward et al., 2012; Vinardell et al., 2012a; Steward et al., 2013), but it would appear that the application of physiological levels (3-10 MPa) of intermittent HP can enhance proteoglygan and collagen synthesis and upregulate aggrecan and collagen II mRNA expression in chondrocytes and stem/progenitor cells isolated from bone marrow, synovial tissues and adipose tissues. There is also evidence to suggest that dynamic HP can act to stabilise the phenotype of chondrogenically primed joint tissue derived MSCs (Vinardell et al., 2012a). A better understanding of how mechanical loading influences terminal differentiation of different tissue-specific MSCs should facilitate efforts to tissue engineer phenotypically stable cartilaginous grafts using such cells.

While an extensive body of work exists comparing the biochemically driven differentiation of MSCs isolated from different tissues, little is known about how these unique cell populations will respond to biophysical stimuli. The objective of this study was to compare how chondrogenically primed BMSCs and FPSCs respond to the application of physiological levels of HP. In particular, we were interested in determining how such joint-specific biomechanical loading influences the stability and functional development of cartilage grafts engineered using cells from both sources. Our hypothesis was that the application of cyclic HP will (i) enhance the functional development of cartilage tissues engineered using both BMSCs and FPSCs, (ii) reduce hypertrophic differentiation and endochondral ossification of cartilaginous grafts engineered using BMSCs and (iii) stabilise and maintain the chondrogenic phenotype in cartilaginous grafts engineered using FPSCs. To mimic the endochondral phenotype observed in tissues engineered using BMSCs, the culture media was supplemented with hypertrophic factors (Sheehy et al., 2012b) and the constructs were simultaneously subjected to $10 \mathrm{MPa}$ of cyclic HP. To mimic the fibrous dedifferentiation observed in tissues engineered using FPSCs, the chondrogenic growth factor transforming growth factor (TGF)- $\beta 3$ was withdrawn from the culture medium (Vinardell et al., 2012b) and again constructs were subjected to $10 \mathrm{MPa}$ of cyclic HP. In this way, it was possible to mimic how physiological loading can impact the phenotypic stability of tissue engineered cartilaginous grafts using a well-controlled in vitro model.

\section{Methods}

\subsection{Agarose gel constructs fabrication and culture}

BMSC and FPSCs were isolated and expanded as described in the Supplementary material section. Both cell types were mixed with $4 \%$ agarose (Type VII; Sigma-Aldrich, Ireland) at a ratio of $1: 1$ at $\sim 40{ }^{\circ} \mathrm{C}$ to yield a final gel concentration of $2 \%$ at a density of 20 million cells $/ \mathrm{mL}$. The agarose-cell constructs $(\varnothing 5 \times 3 \mathrm{~mm})$ were encapsulated within an acellular agarose block (as described below) and maintained in a chemically defined chondrogenic medium consisting of high glucose DMEM supplemented with $100 \mathrm{U} / \mathrm{mL}$ penicillin/streptomycin (both from Gibco), $100 \mathrm{mg} / \mathrm{mL}$ sodium pyruvate, $40 \mathrm{mg} / \mathrm{mL}$ L-proline, $50 \mathrm{mg} / \mathrm{mL}$ L-ascorbic acid-2-phosphate, $1.5 \mathrm{mg} / \mathrm{mL}$ bovine serum albumin, $1 \times$ insulin-transferrin-selenium, $100 \mathrm{nM}$ dexamethasone (all Sigma-Aldrich) and $10 \mathrm{ng} / \mathrm{mL}$ of transforming growth factor- $\beta 3$ (TGF- $\beta 3$, ProSpec-Tany TechnoGene, Ltd.). On day 21 , selected BMSC groups were exposed to hypertrophic medium for 14 days, by changing the following parameters: TGF- $\beta 3$ was withdrawn from the medium, which was then supplemented with $1 \mathrm{nM}$ L-thyroxine (Sigma-Aldrich) and $20 \mu \mathrm{g} \mathrm{ml}^{-1}$ $\beta$-glycerophosphate (Sigma-Aldrich), and the level of dexamethasone was reduced to $1 \mathrm{nM}$. Also, on day 21, additional FPSC groups were maintained in chondrogenic medium (as described above) without TGF- $\beta 3$ for 14 days. Constructs were allowed to equilibrate overnight before the initiation of hydrostatic pressure.

\subsection{Application of hydrostatic pressure}

Cell seeded agarose constructs were encapsulated, evenly spaced, in an agarose block to improve homogeneity of oxygen and nutrient availability and to provide protection from handling damage when the constructs were being placed into and out of the HP bioreactor. Constructs were then inserted into heat-sealed, gaspermeable, water-tight, sterile bags (EVO120, Quest Biomedical, UK) with $4 \mathrm{~mL}$ of medium per construct and the air was removed via a needle free port. Cyclic HP was applied via a water filled, custom-made bioreactor within a $37{ }^{\circ} \mathrm{C}$ incubator as described previously (Meyer et al., 2011). The sealed bags exposed to HP were placed into the pressure vessel, while the free swelling controls were placed into an open water bath next to the pressure vessel. HP was applied at an amplitude of $10 \mathrm{MPa}$ and a frequency of $1 \mathrm{~Hz}$ for a duration of $4 \mathrm{~h}$ per day, 5 days per week for 5 weeks. The bags were returned to a culture incubator $\left(37^{\circ} \mathrm{C}, 5 \% \mathrm{CO}_{2}\right)$ between loading periods and suspended separately in an upright position for homogenous gas transfer. Media changes were performed twice weekly via the needle free port.

\subsection{Mechanical, histological and biochemical assessment of tissues}

The mechanical properties of the engineered constructs were determined as described previously (Buckley and Kelly, 2012). Stress relaxation tests were performed to determine the equilibrium modulus of the tissue, which consisted of a ramp and hold cycle, with a ramp displacement of $1 \mu \mathrm{m} / \mathrm{s}$ until $10 \%$ strain was obtained and then maintained until equilibrium was reached. Dynamic tests, which involved applying a cyclic strain of $1 \%$ at a frequency of $1 \mathrm{~Hz}$, were performed immediately after the stress relaxation cycle to determine the dynamic modulus of the tissue. Constructs were also analysed biochemically to determine their DNA, sulfated glycosaminoglycan (sGAG), collagen and calcium content. Sections were stained with Alcian Blue for sGAG, Picro-sirius Red for collagen and Alizarin Red for mineral deposition, while the localised deposition of collagen types I and II was identified through immunohistochemistry. See Supplementary material section for further details of biochemical and histological assays/methodologies.

\subsection{Experimental design}

To test our hypotheses, three studies were performed. In study 1, BMSC and FPSC seeded constructs were cultured in chondrogenic medium for 5 weeks. In study 2, BMSC constructs were cultured in chondrogenic medium for 3 weeks, followed by 2 weeks culture in hypertrophic medium. In study 3, FPSC constructs were cultured in chondrogenic medium containing TGF- $\beta 3$ for 3 weeks, followed by 2 weeks culture in chondrogenic medium in the presence or absence of TGF- $\beta 3$. All study groups consisted of a hydrostatically loaded group (HP) and a free-swelling unloaded control (FS).

\subsection{Statistical analysis}

All statistical analyses were carried out using Minitab 15.1. Results are reported as mean \pm standard deviation. Groups were analysed using a general linear model for analysis of variance. Tukey's test was used to compare conditions. Significance was accepted at a level of $P<0.05$.

\section{Results}

3.1. Dynamic hydrostatic pressure enhances the functional development of cartilage tissue engineered using both BMSCs and FPSCS

BMSC and FPSC seeded constructs were cultured for 5 weeks in chondrogenic medium in the presence or absence of HP. The application of HP had no effect on the DNA content of either BMSC or FPSC seeded constructs (data not shown). HP significantly 
enhanced SGAG accumulation in both BMSC $(P<0.0001)$ and FPSC $(P<0.0006)$ seeded hydrogels (Fig. 1a and $b)$, but only increased collagen accumulation in BMSC seeded constructs $(P<0.0001$, Fig. 1c and d). Histological staining (Fig. 1e) revealed a high degree of spatial symmetry in ECM deposition for all groups. Alcian Blue staining revealed greater SGAG accumulation in the pericellular region of BMSC constructs (Fig. 1e). sGAG accumulation was more diffuse in FPSC seeded constructs and, at the tissue level, more a

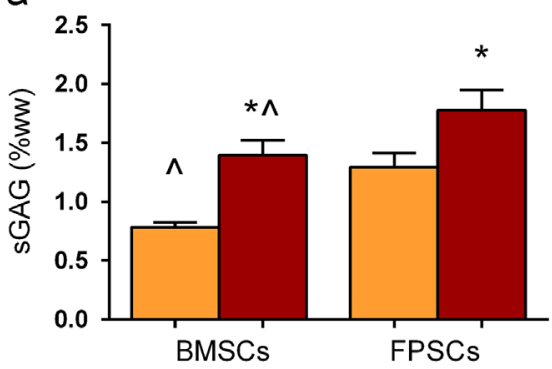

C

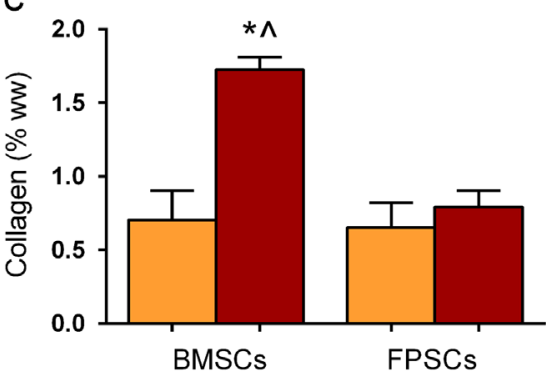

Free Swelling b

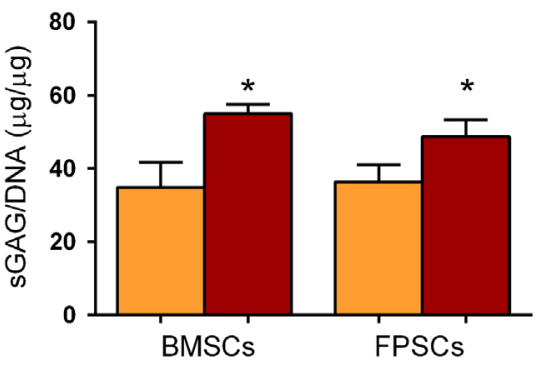

d

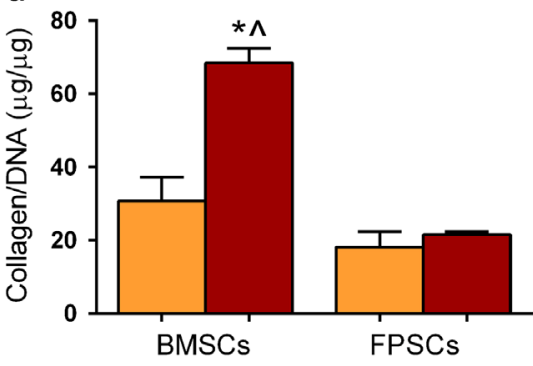

Hydrostatic Pressure e

Bone marrow

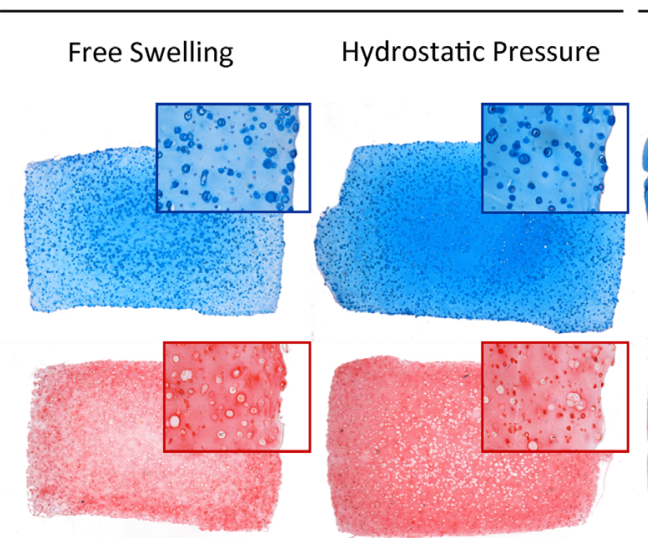

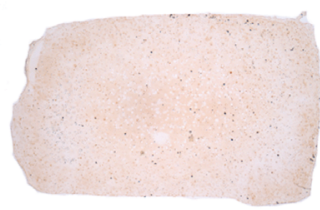
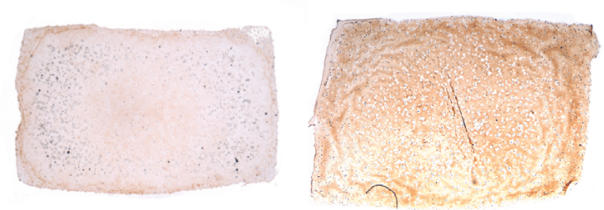
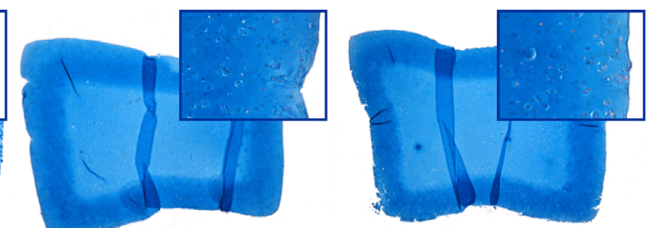

Alcian Blue
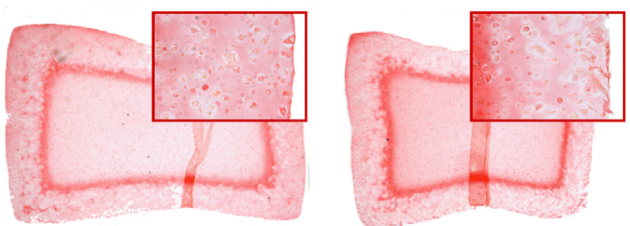

Picrosirius

Red
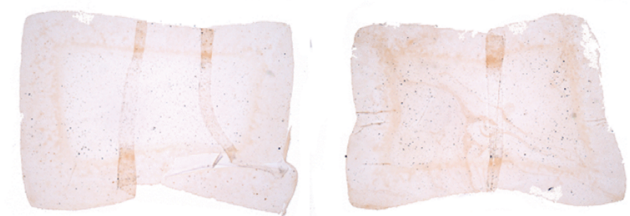

Collagen

Type I
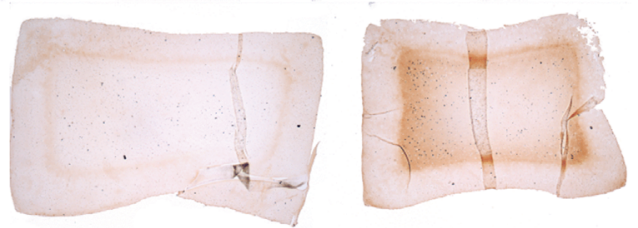

Collagen

Type II

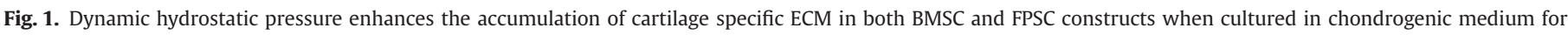

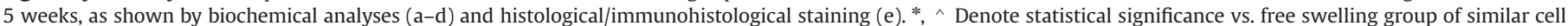
type and vs. FPSCs under similar loading conditions respectively $(P<0.002)$. 
localised to the peripheral region of the engineered tissue. For both cell types, application of HP led to more intense Alcian Blue staining, with more intense Picrosirius Red staining observed in BMSC constructs. While HP had little influence on collagen type I deposition, collagen type II accumulation was enhanced throughout BMSC constructs when exposed to HP. Although FPSC constructs followed a similar trend, staining for collagen II was less intense than BMSC seeded constructs. Increases in cartilage specific ECM accumulation were accompanied by improvements in the functional properties of both BMSC and FPSC derived grafts, as evidenced by significantly increased equilibrium and dynamic moduli following application of hydrostatic pressure (Fig. 2).

\subsection{Dynamic hydrostatic pressure suppresses calcification of cartilage grafts engineered using BMSCS}

The application of HP resulted in significantly $(P<0.002)$ reduced calcium deposition within BMSC seeded constructs maintained in chondrogenic medium (Fig. 3a). This result was confirmed by Alizarin Red staining (Fig. 3b), which showed dramatically reduced staining in the constructs exposed to HP. Next, chondrogenically primed BM constructs were cultured in the presence of hypertrophic factors in order to accelerate the onset and progression of endochondral ossification. No difference was observed in calcium content between the loaded and FS constructs (Fig. 4a), with the addition of hypertrophic factors leading to a decrease in cell viability and a large increase in calcium deposition on a per cell basis. Alizarin Red staining (Fig. 4b), which was restricted to the peripheral region of these constructs, appeared slightly less intense in the loaded constructs.

\subsection{Dynamic hydrostatic pressure acts to maintain a chondrogenic} phenotype in cartilaginous grafts engineered using FPSCS

No evidence of calcium deposition was observed in cartilaginous tissues engineered using FPSCs (data not shown). To mimic the fibrous dedifferentiation observed in cartilaginous grafts engineered using FPSCs following subcutaneous implantation (Vinardell et al., 2012b), constructs were cultured for 3 weeks in chondrogenic medium containing TGF- $\beta 3$, followed by 2 weeks where TGF- $\beta 3$ was withdrawn from the culture media of half of the groups, in the presence or absence of HP. The withdrawal of TGF- $\beta 3$ from FPSC seeded constructs after 3 weeks (FS-) had no effect on the DNA content of the engineered tissues (Fig. 5a), when compared to continuous TGF- $\beta 3$ supplementation (FS). However, TGF- $\beta 3$ withdrawal resulted in a significant decrease in SGAG and collagen deposition by week 5 . Interestingly, in the absence of TGF- $\beta 3$, the application of hydrostatic pressure (HP-) partially mitigated this reduction, with regards to sGAG synthesis, and fully restored collagen levels to that observed in the non-loaded, TGF- $\beta 3$ supplemented constructs. Histological staining (Fig. 5b) demonstrated greater levels of sGAG and collagen accumulation in the loaded group when compared to the free swelling group, with a more homogenous distribution of extracellular matrix (ECM) throughout the construct.

\section{Discussion}

Understanding how joint specific environmental factors regulate the phenotype of MSCs is central to developing novel cell based therapies for cartilage regeneration. In this study, we utilised a HP bioreactor to firstly elucidate how this stimulus can be used to enhance the functional development of cartilage constructs engineered using multipotent cells derived from different tissue sources, and secondly, as a model system to understand how joint specific environmental factors such as HP will regulate phenotypic stability of such cartilage grafts. In agreement with previous studies (Meyer et al., 2011), the application of HP was found to enhance the mechanical functionality of cartilaginous grafts engineered using BMSCs and, furthermore, to suppress the inherent tendency of these tissues to mineralise and progress

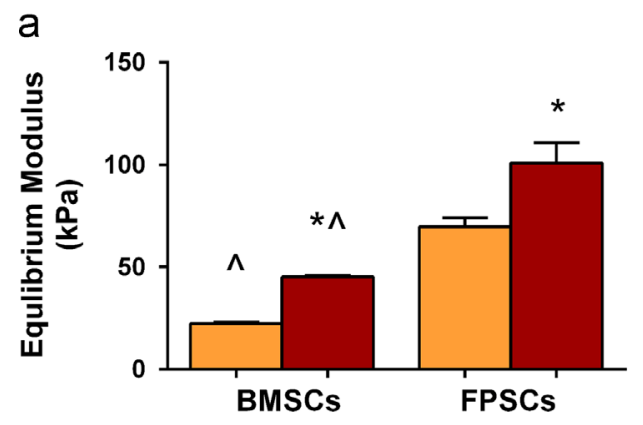

Free Swelling

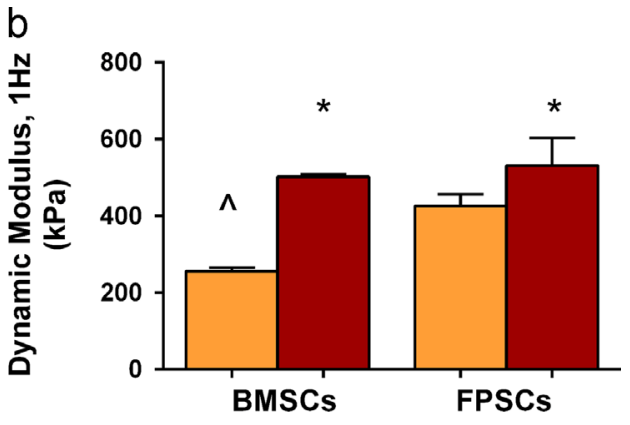

Hydrostatic Pressure

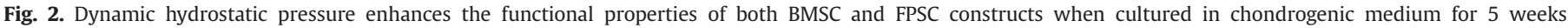
$*$, $\wedge$ Denote statistical significance vs. free swelling group of similar cell type and vs. FPSCs under similar loading conditions respectively $(P<0.002)$.

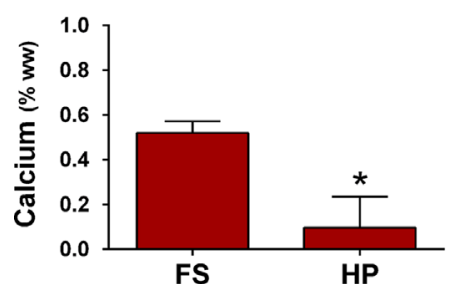

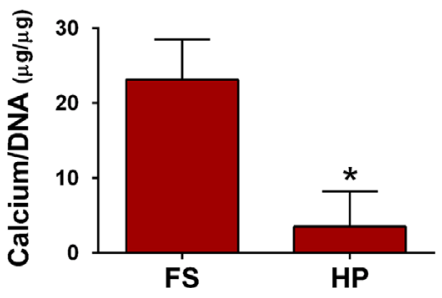

b

FS

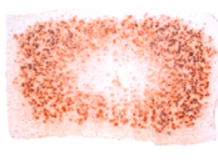

HP

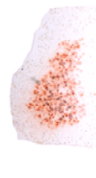

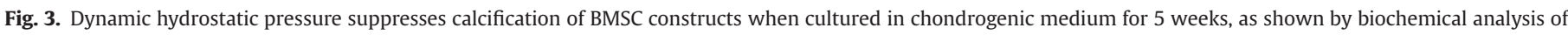

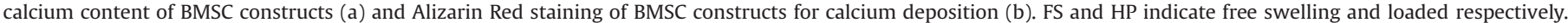

* Denotes statistical significance vs. free swelling group of similar cell type $(P<0.002)$. 
a

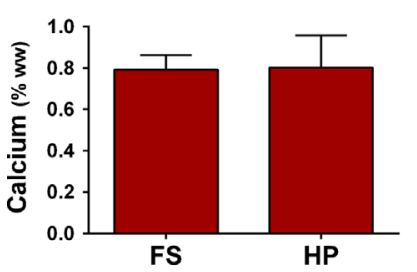

b

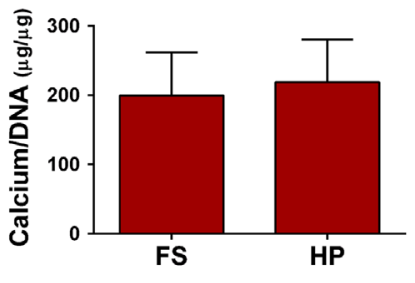

FS

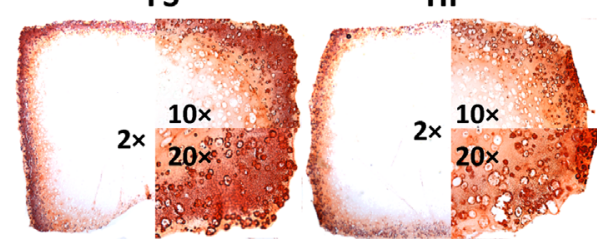

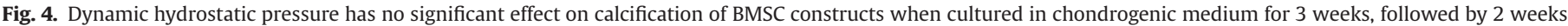

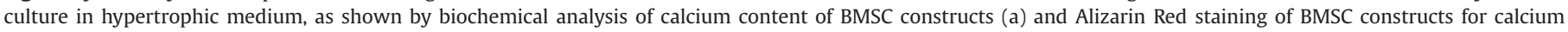
deposition (b). FS and HP indicate free swelling and loaded respectively. Original image magnification is shown inset.

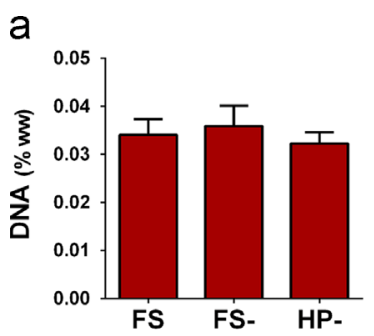

b
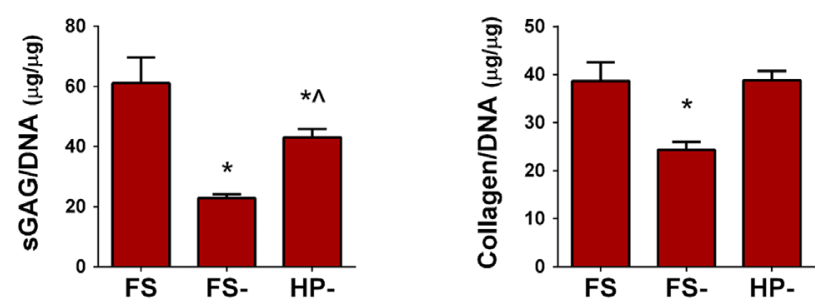

FS
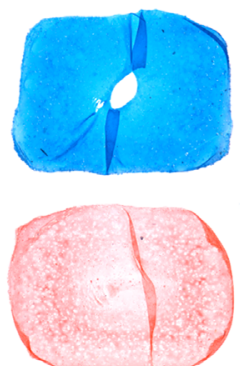

FS -
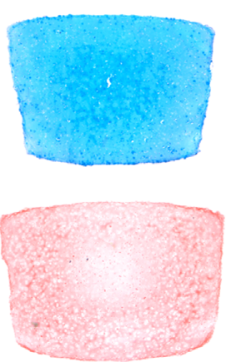

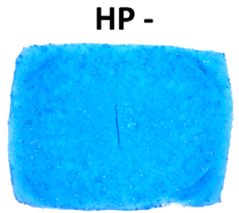

Alcian

Blue

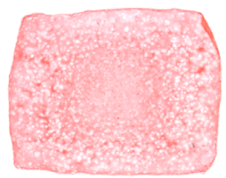

Red

Picrosirius

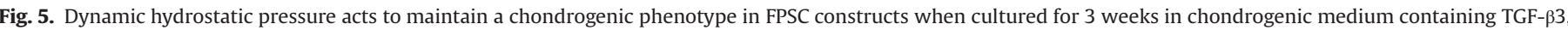

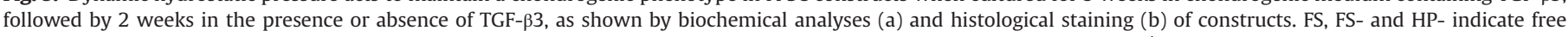

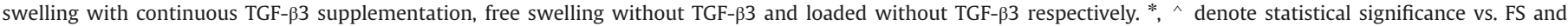
FS- groups respectively $(P<0.001)$.

along the endochondral route. In addition, HP would also appear to play a role in maintaining the chondrogenic phenotype of FPSCs, as indicated by the findings that, upon removal of TGF- $\beta 3$, HP also acted to enhance cartilage-specific extracellular matrix development. These results suggest that cartilaginous tissues engineered using either cell source have the ability to terminally differentiate towards a stable chondrogenic phenotype within an environment where the constructs are subjected to high magnitude cyclic HP.

This study adds to the growing body of literature pointing to the beneficial effects of mechanical stimuli, which appear to improve the functionality of cartilage grafts engineered using MSCs. Articular cartilage is a multiphasic tissue, the solid phase of which is composed predominately of a collagen (mainly type II) fibrillar network (around 10-22\% by wet weight) enmeshing proteoglycan macromolecules (2-7\% by wet weight) (Maroudas et al., 1969; Muir et al., 1970; Bayliss and Ali, 1978; Inerot et al., 1978; Maroudas et al., 1980; Zirn et al., 1984; Mow et al., 1992; Rieppo et al., 2009; Gannon et al., 2012). Previous studies have demonstrated that the application of dynamic compression (Huang et al., 2010; Bian et al., 2012; Thorpe et al., 2013) or hydrostatic pressure (Miyanishi et al., 2006; Wagner et al., 2008; Ogawa et al., 2009; Huang et al., 2010; Meyer et al., 2011; Correia et al., 2012; Liu et al., 2012; Steward et al., 2012; Vinardell et al., 2012a; Liu et al., 2013) can enhance the sGAG and collagen content as well as the mechanical functionality of cartilage constructs engineered using MSCs. However, these studies often fail to produce cartilage grafts with mechanical functionality or ECM content approaching that of native cartilage. It appears that the efficacy of pre-implantation mechanical stimulation is dependent on many variables, such as substrate stiffness (Steward et al., 2013, in press), magnitude and duration of applied loading (Ikenoue et al., 2003; Correia et al., 2012), time of initiation of loading during culture period (Thorpe et al., 2010), cell donor source (Meyer et al., 2011) and, as demonstrated in the current study, the tissue source of MSCs. It remains to be determined how mechanically functional a tissue needs to be to ensure regeneration of hyaline tissue, although computational studies suggest the likelihood of success will improve with increases in preimplantation functionality (Nagel and Kelly, 2013).

The mean equilibrium modulus of FPSC constructs was found to be greater than that of BMSC constructs. This can be attributed, at least in part, to the higher levels of SGAG accumulation (as a percentage of wet weight) within FPSC constructs. The differences in spatial matrix accumulation may also play a role in determining the apparent mechanical properties of the engineered tissues (Kelly and Prendergast, 2004; Khoshgoftar et al., 2012). Following the application of HP, there was no difference in the dynamic modulus of BMSC and FPSC constructs. This can be attributed to the large increase in collagen deposition observed in BMSC constructs following the application of HP. While the equilibrium 
modulus of engineered cartilage is known to be strongly correlated to the sGAG content (Kelly et al., 2007; Vinardell et al., 2011), the dynamic modulus also strongly depends on the functionality of the collagen network, as it plays a key role in enabling the tissue to generate fluid load support (Ateshian, 2009).

Within full thickness cartilage defects, it has been demonstrated that hypertrophy of implanted MSCs is restricted to a region of the regenerating tissue close to the bone, suggesting that biomechanical stimuli play a key role in determining the stability of the chondrogenic phenotype (Steck et al., 2009). The results of this study support this hypothesis, with the application of HP found to dramatically reduce the mineralisation of BMSC constructs cultured in a chondro-inductive medium. This effect was not observed, however, when constructs were maintained in medium supplemented with hypertrophic factors. A possible reason for this is that these factors may provide an unphysiological, potent chemical cue that mechanical stimulation cannot override. The in vivo mechanical environment is complex, and MSCs will not only experience high levels of HP, but also shape changing deformations as the tissue is subjected to tension and compression during normal joint loading. Indeed, previous studies in our lab and others have demonstrated that other such mechanical cues, such as dynamic compression, can also suppress hypertrophy of MSCs (Bian et al., 2012; Thorpe et al., 2013). In addition to mechanical loading, MSCs in the joint defect environment will also experience other phenotype defining stimuli, such as hypoxic conditions, which have also been shown to suppress hypertrophy and endochondral ossification of chondrogenically primed MSCs (Sheehy et al., 2012a). Understanding how MSCs integrate the multiple mechanical and biochemical cues they will experience in vivo and how these cues direct their phenotype is a central challenge in the field of regenerative medicine.

The mechanism by which HP enhances cartilage-specific matrix synthesis and suppresses hypertrophy of MSCs has not yet been fully elucidated. According to the biphasic theory for cartilage, the solid matrix of the tissue can be considered to be intrinsically incompressible when loaded within the normal physiologic range of pressures and, unlike with dynamic compression, tissue deformation will be minimal under pure hydrostatic pressurisation (Bachrach et al., 1998). Because of this, alternative mechanisms of HP mechanotransduction have been explored. There is evidence to suggest that HP affects the cell membrane ion channels (Browning et al., 1999), possibly via changes in the conformations of the transmembrane proteins (Kornblatt and Kornblatt, 2002). The resulting changes in intracellular ion concentrations can then, in turn, result in changes in cellular gene expression and protein synthesis (Horowitz and Lau, 1988). Several studies have also indicated that HP can trigger the release of intracellular calcium stores (Browning et al., 2004; Mizuno, 2005), but further investigation will be necessary to determine whether such factors regulate the onset of hypertrophy in BMSCs. What has been demonstrated is that a stiffer substrate promotes a more endochondral phenotype in BMSCs (Bian et al., 2013). Since the pericellular environment will become stiffer as ECM content increases with time in culture, this may contribute to the development of an endochondral phenotype in BMSC constructs. However, it has also been shown that HP can override the influence of a stiffening microenvironment on chondrogenesis of MSCs (Steward et al., 2013), possibly by modulating changes to the cytoskeleton. Taken together, these results raise the possibility that HP may act to suppress a substrate stiffness mediated progression of hypertrophy in BMSCs, although further work is clearly required to directly test this hypothesis.

In conclusion, the results of this study demonstrate that the application of physiological levels of HP to cartilage grafts engineered using different types of MSCs not only improves their mechanical functionality, but also leads to the development of a more stable cartilaginous phenotype. A better understanding of stem cell mechanobiology should open up many exciting new possibilities for the field of articular cartilage tissue engineering and regenerative medicine. It may allow for the development of in vitro bioreactor stimulation protocols to engineer not only more mechanically functional grafts, but also tissues that are resistant to hypertrophy and endochondral ossification. Perhaps more importantly, a more complete understanding of stem cell mechanobiology may ultimately allow us to manipulate the in vivo mechanical environment (for example, by modulating the mechanical properties of scaffolds/engineered tissues or through appropriate postoperative rehabilitation regimes) to ensure such cells terminally differentiate towards a desired phenotype upon implantation into the body.

\section{Conflict of interest statement}

The authors have no conflicts of interest to disclose.

\section{Acknowledgements}

Funding was provided by Science Foundation Ireland (President of Ireland Young Researcher Award: 08/YI5/B1336) and the European Research Council (StemRepair - Project number 258463).

\section{Appendix A. Supplementary material}

Supplementary data associated with this article can be found in the online version at http://dx.doi.org/10.1016/j.jbiomech.2013.12.006.

\section{References}

Ateshian, G.A., 2009. The role of interstitial fluid pressurisation in articular cartilage lubrication. J. Biomech. 42 (9), 1163-1176.

Bachrach, N.M., Mow, V.C., Guilak, F., 1998. Incompressibility of the solid matrix of articular cartilage under high hydrostatic pressures. J. Biomech. 31 (5), 445-451.

Bayliss, M.T., Ali, S.Y., 1978. Age-related changes in the composition and structure of human articular-cartilage proteoglycans. Biochem. J. 176, 683-693.

Bian, L., Hou, C., Tous, E., Rai, R., Mauck, R.L., Burdick, J.A., 2013. The influence of hyaluronic acid hydrogel crosslinking density and macromolecular diffusivity on human MSC chondrogenesis and hypertrophy. Biomaterials 34 (2), 412-421.

Bian, L., Zhai, D.Y., Zhang, E.C., Mauck, R.L., Burdick, J.A., 2012. Dynamic compressive loading enhances cartilage matrix synthesis and distribution and suppresses hypertrophy in hMSC-laden hyaluronic acid hydrogels. Tissue Eng. Part A 18 (7-8), 715-724.

Browning, J., Walker, R., Hall, A., Wilkins, R., 1999. Modulation of $\mathrm{Na}+\mathrm{H}+$ exchange by hydrostatic pressure in isolated bovine articular chondrocytes. Acta Physiol Scand. 166 (1), 39-45.

Browning JA Saunders, K. Urban, JP, Wilkins, RJ, 2004. The influence and interactions of hydrostatic and osmotic pressures on the intracellular milieu of chondrocytes. Biorheology 41 (3-4), 299-308.

Bruder, S.P., Jaiswal, N., Haynesworth, S.E., 1997. Growth kinetics, self-renewal, and the osteogenic potential of purified human mesenchymal stem cells during extensive subcultivation and following cryopreservation. J. Cell. Biochem. 64 (2), 278-294.

Buckley, CT. Kelly, DJ. 2012. Expansion in the presence of FGF-2 enhances the functional development of cartilaginous tissues engineered using infrapatellar fat pad derived MSCs. J. Mech. Behav. Biomed. Mater. 11, 102-111.

Caplan, A.I., 1991. Mesenchymal stem cells. J. Orthop. Res. 9 (5), 641-650.

Carver, S.E., Heath, C.A., 1999. Semi-continuous perfusion system for delivering intermittent physiological pressure to regenerating cartilage. Tissue Eng. 5 (1) $1-11$.

Carver, S.E. Heath, C.A. 2000. Increasing extracellular matrix production in regenerating cartilage with intermittent physiological pressure. Biotechnol. Bioeng. 62 (2), 166-174.

Correia, C., Pereira, A.L., Duarte, A.R., Frias, A.M., Pedro, A.J., Oliveira, J.T., Sousa, R.A. Reis, R.L., 2012. Dynamic culturing of cartilage tissue: the significance of hydrostatic pressure. Tissue Eng. - Part A 18 (19-20), 1979-1991.

De Bari, C., Dell'Accio, F., Luyten, F.P., 2004. Failure of in vitro-differentiated mesenchymal stem cells from the synovial membrane to form ectopic stable cartilage in vivo. Arthritis Rheum. 50 (1), 142-150. 
Dickhut, A., Gottwald, E., Steck, E., Heisel, C., Richter, W., 2008. Chondrogenesis of mesenchymal stem cells in gel-like biomaterials in vitro and in vivo. Front. Biosc. 13, 4517-4528.

Finger, A.R., Sargent, C.Y., Dulaney, K.O., Bernacki, S.H., Loboa, E.G., 2007. Differential effects on messenger ribonucleic acid expression by bone marrow-derived human mesenchymal stem cells seeded in agarose constructs due to ramped and steady applications of cyclic hydrostatic pressure. Tissue Eng. 13 (6), 1151-1158.

Gannon, A.R., Nagel, T., Kelly, D.J., 2012. The role of the superficial region in determining the dynamic properties of articular cartilage. Osteoarthr. Cartil..

Hennig, T., Lorenz, H., Thiel, A., Goetzke, K., Dickhut, A., Geiger, F., Richter, W., 2007 Reduced chondrogenic potential of adipose tissue derived stromal cells correlates with an altered TGFbeta receptor and BMP profile and is overcome by BMP-6. J. Cell. Physiol. 211 (3), 682-691.

Horowitz, S.B., Lau, Y.T., 1988. A function that relates protein synthetic rates to potassium activity in vivo. J. Cell. Physiol. 135 (3), 425-434.

Hu, J.C., Athanasiou, K.A., 2006. The effects of intermittent hydrostatic pressure on self-assembled articular cartilage constructs. Tissue Eng. 12 (5), 1337-1344.

Huang, A.H., Farrell, M.J., Kim, M., Mauck, R.L., 2010. Long-term dynamic loading improves the mechanical properties of chondrogenic mesenchymal stem cellladen hydrogels. Eur. Cells Mater. 19, 72-85.

Ikenoue, T., Trindade, M.C., Lee, M.S., Lin, E.Y., Schurman, D.J., Goodman, S.B., Smith, R.L., 2003. Mechanoregulation of human articular chondrocyte aggrecan and type II collagen expression by intermittent hydrostatic pressure in vitro. J. Orthop. Res. 21 (1), 110-116.

Inerot, S., Heinegard, D., Audell, L., Olsson, S., 1978. Articular-cartilage proteoglycans in aging and osteoarthritis. Biochem. J. 169, 143-156.

Johnstone, B., Hering, T.M., Caplan, A.I., Goldberg, V.M., Yoo, J.U., 1998. In vitro chondrogenesis of bone marrow-derived mesenchymal progenitor cells. Exp. Cell Res. 238 (1) 265-272.

Jortikka, M.O., Parkkinen, J.J., Inkinen, R.I., Karner, J., Jarvelainen, H.T., Nelimarkka, L. O., Tammi, M.I., Lammi, M.J., 2000. The role of microtubules in the regulation of proteoglycan synthesis in chondrocytes under hydrostatic pressure. Arch. Biochem. Biophys. 374 (2), 172-180.

Kadiyala, S., Young, R.G., Thiede, M.A., Bruder, S.P., 1997. Culture expanded canine mesenchymal stem cells possess osteochondrogenic potential in vivo and in vitro. Cell Transplant. 6 (2), 125-134.

Kelly, D., Prendergast, P.J., 2004. Effect of a degraded core on the mechanical behaviour of tissueengineered cartilage constructs: a poro-elastic finite element analysis. Med. Biol. Eng. Comput. 42 (1), 9-13.

Kelly, D.J., Crawford, A., Dickinson, S.C., Sims, T.J., Mundy, J., Hollander, A.P., Prendergast, P.J., Hatton, P.V., 2007. Biochemical markers of the mechanical quality of engineered hyaline cartilage. J. Mater. Sci.: Mater. Med. 18 (2), 273-281.

Khoshgoftar, M., Wilson, W., Ito, K., van Donkelaar, C.C., 2012. Influence of tissueand cell-scale extracellular matrix distribution on the mechanical properties of tissue-engineered cartilage. Biomech. Model. Mechanobiol., 1-13.

Kornblatt, J.A., Kornblatt, M.J., 2002. The effects of osmotic and hydrostatic pressures on macromolecular systems. Biochim. Biophys. Acta 1595 (1-2), 30-47.

Liu, Y., Buckley, C.T., Downey, R., Mulhall, K.J., Kelly, D.J., 2012. The role of environmental factors in regulating the development of cartilaginous grafts engineered using osteoarthritic human infrapatellar fat pad-derived stem cells. Tissue Eng. Part A.

Liu, Y., Buckley, C.T., Mulhall, K.J., Kelly, D.J., 2013. Combining BMP-6, TGF- $\beta 3$ and hydrostatic pressure stimulation enhances the functional development of cartilage tissues engineered using human infrapatellar fat pad derived stem cells. Biomater. Sci.

Mackay, A.M., Beck, S.C., Murphy, J.M., Barry, F.P., Chichester, C.O., Pittenger, M.F., 1998. Chondrogenic differentiation of cultured human mesenchymal stem cells from marrow. Tissue Eng. 4 (4), 415-428.

Maroudas, A., Bayliss, M., Venn, M., 1980. Further studies on the composition of human femoral head cartilage. Ann. Rheum. Dis. 39 (5), 514-523.

Maroudas, A., Muir, H., Wingham, J., 1969. The correlation of fixed negative charge with glycosaminoglycan content of human articular cartilage. Biochim. Biophys. Acta (BBA) - Gen. Subj. 177 (3), 492-500.

Maxson, S., Burg, K.J., 2012. Synergistic effects of conditioned media and hydrostatic pressure on the differentiation of mesenchymal stem cells. Cell. Mol. Bioeng. 5 (4), 414-426.

Meyer, E.G., Buckley, C.T., Steward, A.J., Kelly, D.J., 2011. The effect of cyclic hydrostatic pressure on the functional development of cartilaginous tissues

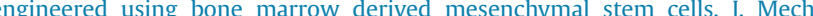
Behav. Biomed. Mater. 4 (7), 1257-1265.

Miyanishi, K., Trindade, M.C., Lindsey, D.P., Beaupre, G.S., Carter, D.R., Goodman, S.B., Schurman, D.J., Smith, R.L., 2006. Effects of hydrostatic pressure and transforming growth factor-beta 3 on adult human mesenchymal stem cell chondrogenesis in vitro. Tissue Eng. 12 (6), 1419-1428.

Mizuno, S., 2005. A novel method for assessing effects of hydrostatic fluid pressure on intracellular calcium: a study with bovine articular chondrocytes. Am. J. Physiol. - Cell Physiol. 288 (2), C329-C337.

Mow, V.C., Ratcliffe, A., Robin Poole, A., 1992. Cartilage and diarthrodial joints as paradigms for hierarchical materials and structures. Biomaterials 13 (2), 67-97.

Muir, H., Bullough, P., Maroudas, A., 1970. The distribution of collagen in human articular cartilage with some of its physiological implications. J. Bone Jt. Surg. Br. 52 (3), 554-563.

Nagel, T., Kelly, D.J., 2013. The composition of engineered cartilage at the time of implantation determines the likelihood of regenerating tissue with a normal collagen architecture. Tissue Eng. - Part A 19 (7-8), 824-833.
Ogawa, R., Mizuno, S., Murphy, G.F., Orgill, D.P., 2009. The effect of hydrostatic pressure on three-dimensional chondroinduction of human adipose-derived stem cells. Tissue Eng. - Part A 15 (10), 2937-2945.

Parkkinen, J.J., Ikonen, J., Lammi, M.J., Laakkonen, J., Tammi, M., Helminen, H.J., 1993. Effects of cyclic hydrostatic pressure on proteoglycan synthesis in cultured chondrocytes and articular cartilage explants. Arch. Biochem. Biophys. 300 (1), 458-465.

Pelttari, K., Winter, A., Steck, E., Goetzke, K., Hennig, T., Ochs, B.G., Aigner, T., Richter, W., 2006. Premature induction of hypertrophy during in vitro chondrogenesis of human mesenchymal stem cells correlates with calcification and vascular invasion after ectopic transplantation in SCID mice. Arthritis Rheum. 54 (10), 3254-3266.

Pittenger, M.F., Mackay, A.M., Beck, S.C., Jaiswal, R.K., Douglas, R., Mosca, J.D., Moorman, M.A., Simonetti, D.W., Craig, S., Marshak, D.R., 1999. Multilineage potential of adult human mesenchymal stem cells. Science 284 (5411), 143-147.

Puetzer, J., Williams, J., Gillies, A., Bernacki, S., Loboa, E.G. 2012. The effects of cyclic hydrostatic pressure on chondrogenesis and viability of human adipose-and bone marrow-derived mesenchymal stem cells in three-dimensional agarose constructs. Tissue Eng. Part A 19 (1-2), 299-306.

Rieppo, J., Hyttinen, M.M., Halmesmaki, E., Ruotsalainen, H., Vasara, A., Kiviranta, I. Jurvelin, J.S., Helminen, H.J., 2009. Changes in spatial collagen content and collagen network architecture in porcine articular cartilage during growth and maturation. Osteoarthr. Cartil. 17 (4), 448-455.

Safshekan, F., Tafazzoli-Shadpour, M., Shokrgozar, M.A., Haghighipour, N., Mahdian, R., Hemmati, A., 2012. Intermittent hydrostatic pressure enhances growth factor-induced chondroinduction of human adipose-derived mesenchymal stem cells. Artif. Organs 36 (12), 1065-1071.

Sakaguchi, Y., Sekiya, I., Yagishita, K., Muneta, T., 2005. Comparison of human stem cells derived from various mesenchymal tissues - Superiority of synovium as a cell source. Arthritis Rheum. 52 (8), 2521-2529.

Segawa, Y., Muneta, T., Makino, H., Nimura, A., Mochizuki, T., Ju, Y.J., Ezura, Y., Umezawa, A., Sekiya, I., 2009. Mesenchymal stem cells derived from synovium, meniscus, anterior cruciate ligament, and articular chondrocytes share similar gene expression profiles. J. Orthop. Res. 27 (4), 435-441.

Sheehy, E.J., Buckley, C.T., Kelly, D.J., 2012a. Oxygen tension regulates the osteogenic, chondrogenic and endochondral phenotype of bone marrow derived mesenchymal stem cells. Biochem. Biophys. Res. Commun. 417 (1), 305-310.

Sheehy, E.J., Vinardell, T., Buckley, C.T., Kelly, D.J., 2012b. Engineering osteochondral constructs through spatial regulation of endochondral ossification. Acta Biomater.

Smith, R.L., Goodman, S., Schurman, D., Carter, D., 2000. Time-dependent effects of intermittent hydrostatic pressure on articular condrocyte type II collagen and aggrecan mRNA expression. J. Rehabil. Res. Dev. 37 (2), 153-161.

Smith, R.L., Rusk, S., Ellison, B., Wessells, P., Tsuchiya, K., Carter, D., Caler, W., Sandell, L., Schurman, D., 2005. In vitro stimulation of articular chondrocyte mRNA and extracellular matrix synthesis by hydrostatic pressure. J. Orthop. Res. 14 (1), 53-60.

Steck, E., Fischer, J., Lorenz, H., Gotterbarm, T., Jung, M., Richter, W., 2009. Mesenchymal stem cell differentiation in an experimental cartilage defect: restriction of hypertrophy to bone-close neocartilage. Stem Cells Dev. 18 (7), 969-978.

Steward, A., Wagner, D., Kelly, D., 2013. The pericellular environment regulates cytoskeletal development and the differentiation of mesenchymal stem cells and determines their response to hydrostatic pressure. Eur. Cells Mater. 25, 167.

Steward, A.J., Thorpe, S.D., Vinardell, T., Buckley, C.T., Wagner, D.R., Kelly, D.J., 2012. Cell-matrix interactions regulate mesenchymal stem cell response to hydrostatic pressure. Acta Biomater. 8 (6), 2153-2159.

Steward, Wagner, Kelly, Exploring the roles of integrin binding and cytoskeletal reorganization during mesenchymal stem cell mechanotransduction in soft and stiff hydrogels subjected to dynamic compression, Journal of the Mechanical Behavior of Biomedical Materials, (in press), http://dx.doi.org/10.1016/j. jmbbm.2013.07.020.

Suh, J.K., Baek, G.H., Aroen, A., Malin, C.M., Niyibizi, C., Evans, C.H., Westerhausen-Larson, A., 1999. Intermittent sub-ambient interstitial hydrostatic pressure as a potentia mechanical stimulator for chondrocyte metabolism. Osteoarthr. Cartil. 7 (1), 71-80.

Thorpe, S.D., Buckley, C.T., Vinardell, T., O'Brien, F.J., Campbell, V.A., Kelly, D.J., 2010. The response of bone marrow-derived mesenchymal stem cells to dynamic compression following TGF- $\beta 3$ induced chondrogenic differentiation. Ann. Biomed. Eng. 38 (9), 2896-2909.

Thorpe, S.D., Nagel, T., Carroll, S.F., Kelly, D.J., 2013. Modulating gradients in regulatory signals within mesenchymal stem cell seeded hydrogels: a novel strategy to engineer zonal articular cartilage. PLoS ONE 8, 4

Vinardell, T., Buckley, C.T., Thorpe, S.D., Kelly, D.J., 2011. Composition-function relations of cartilaginous tissues engineered from chondrocytes and mesenchymal stem cells isolated from bone marrow and infrapatellar fat pad. J. Tissue Eng. Regen. Med. 5 (9), 673-683.

Vinardell, T., Rolfe, R.A., Buckley, C.T., Meyer, E.G., Ahearne, M., Murphy, P., Kelly, D.J., 2012a. Hydrostatic pressure acts to stabilise a chondrogenic phenotype in porcine joint tissue derived stem cells. Eur. Cells Mater. 23, 121-132. (discussion 133-124).

Vinardell, T., Sheehy, E.J., Buckley, C.T., Kelly, D.J., 2012b. A comparison of the functionality and in vivo phenotypic stability of cartilaginous tissues engineered from different stem cell sources. Tissue Eng. - Part A 18 (11-12), 1161-1170.

Wagner, D.R., Lindsey, D.P., Li, K.W., Tummala, P., Chandran, S.E., Smith, R.L. Longaker, M.T., Carter, D.R., Beaupre, G.S., 2008. Hydrostatic pressure enhances chondrogenic differentiation of human bone marrow stromal cells in osteochondrogenic medium. Ann. Biomed. Eng. 36 (5), 813-820.

Zirn, J.R., Schurman, D.J., Smith, R.L., 1984. Keratan sulfate content and articular cartilage maturation during postnatal rabbit growth. J. Orthop. Res. 2 (2), $143-150$. 